\section{A) Check for updates}

Cite this: Food Funct., 2020, 11 2040

Received 2nd September 2019, Accepted 4th March 2020

DOI: 10.1039/c9fo02033k

rsc.li/food-function

\title{
Enzymatic cross-linking of pectin in a high-pressure foaming process
}

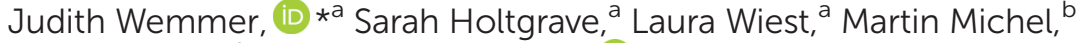 \\ Martin E. Leser ${ }^{b}$ and Erich J. Windhab (D) *a
}

\begin{abstract}
The enzyme laccase is a copper-containing oxidoreductase with the ability to oxidize a wide range of substrates, such as ferulic acid. Thus, the ferulic acid-containing sugar beet pectin (SBP) can be crosslinked through laccase-mediated oxidation. As cross-linking increases viscosity, it could be applied to stabilize SBP-containing foams. In this study, laccase-mediated cross-linking of SBP was investigated under conditions of a high-pressure foaming process. Shear, presence of $\mathrm{CO}_{2}$, and pressure were simulated in a rheometer equipped with a high-pressure cell. At rest, addition of laccase to SBP solution led to the formation of a stiff gel. Application of shear upon mixing of laccase and SBP solution decreased the storage modulus with increasing shear duration and shear rate. This can be attributed to the formation of a fluid gel. However, when shear was stopped before all available ferulic acid groups were cross-linked, a stronger and more coherent network was formed. Pressure exerted by $\mathrm{CO}_{2}$ did not affect cross-linking. Additionally, this approach was tested in a stirred high-pressure vessel where SBP was foamed through $\mathrm{CO}_{2}$ dissolution under pressure and shear followed by controlled pressure release. While pure SBP foam was highly unstable, addition of laccase decelerated collapse. Highest stability was reached when laccase and SBP were mixed prior to depressurization. At the point of foam formation, the continuous phase was thereby viscous enough to increase foam stability. At the same time, continuation of cross-linking at rest caused gel templating of the foam structure.
\end{abstract}

\section{Introduction}

Foaming processes are widely practised to tailor mechanical properties, density and surface area of materials through gas entrapment. As foams are thermodynamically unstable, bubbles will eventually grow and escape the structure, resulting in a loss of desired properties. ${ }^{1}$ The lifetime of a foam is highly dependent on the size and distribution of entrapped bubbles as well as on interfacial and rheological properties of the surrounding material. Shelf-life is often prolonged by increasing viscosity of the continuous phase through thermal processes, e.g., by cooling in extruded snacks, foam-extruded plastics or ice cream, or by heating in baked goods. In soft foamed materials, such as desserts or cosmetics, thickening and gelling agents are added for stabilization. ${ }^{1}$ However, additives might not be desired by consumers who seek for "natural", "clean-label" products. Thus, alternative strategies for structure stabilization such as enzyme-mediated crosslinking are of rising interest. ${ }^{2,3}$

\footnotetext{
${ }^{a}$ Laboratory of Food Process Engineering, ETH Zürich, Schmelzbergstr. 9, 8092 Zurich, Switzerland. E-mail: judith.wemmer@hest.ethz.ch, erich.windhab@hest.ethz.ch

${ }^{b}$ Nestlé Research Lausanne, Vers-chez-les-blanc, CH-1000 Lausanne 26, Switzerland
}

The enzyme laccase is a copper-containing oxidoreductase found in plants, bacteria and insects with the ability to oxidize a wide range of substrates containing polyphenols, methoxysubstituted phenols or diamines. The oxidation results in a free radical, which may take part in a polymerization reaction. ${ }^{4}$ Pectin extracted from sugar beet contains the methoxy-substituted phenol ferulic acid esterified to arabinose or galactose residues. The oxidation of these ferulic acid groups by laccase and the subsequent cross-linking reaction result in covalently cross-linked sugar beet pectin (SBP) causing an increase in viscosity or gelation of SBP in solution. ${ }^{5,6}$ Thus, laccase-mediated cross-linking of SBP can be applied to stabilize multi-phase systems. Littoz et al. $^{7}$ and Zeeb et al. $^{8}$ studied enzymatic crosslinking of interfacial layers around emulsion droplets. Both studies showed improved stability and lower pH-dependency of cross-linked double-layered emulsions stabilized by $\beta$-lactoglobulin and sugar beet pectin ${ }^{7}$ and fish gelatin and sugar beet pectin, ${ }^{8}$ respectively. Soltani and Madadlou ${ }^{9,10}$ investigated the stabilization of fish-oil loaded zein particles suspended in cross-linked pectin gels. They showed that loading with particles led to decelerated laccase-mediated cross-linking of pectin in the continuous phase compared to particle-free pectin solution. ${ }^{9}$ In a second study, laccasemediated cross-linking was supported by subsequent calcium 
addition and led to sufficient gelation of particle-loaded pectin solution through synergistic action of both gelation mediators. ${ }^{10}$

Similarly, this study attempts to increase foam stability of SBP foams through cross-linking of the continuous phase mediated by laccase. This approach is of particular interest for foams generated by dissolution of gas under pressure and shear followed by depressurization, referred to as highpressure foaming. Its application ranges from foaming in whipped cream dispensers to industrial-scale foam extrusion. In this process, a low viscosity of the continuous phase is desired for the step of gas dissolution due to higher diffusion rate and enhanced convection. ${ }^{11,12}$ The addition of enzymes could then trigger an increase in continuous phase viscosity or gelation upon foam generation. In situ cross-linking of foam has been explored for ceramics where catalysts and initiators added after the foaming step facilitate the polymerization reaction of acrylate monomers resulting in fixation. The time for polymerization onset was found to affect bubble size in the fixated foam. ${ }^{13}$ In order to assess possible process time windows for enzyme addition, it is crucial to understand how process conditions of the high-pressure foaming process affect cross-linking.

The objective of this study was to test if and how laccasemediated cross-linking of sugar beet pectin could be integrated in a high-pressure foaming process in order to increase foam stability. Application of shear, pressure and presence of carbon dioxide, relevant for high-pressure foaming, were simulated in a rotational rheometer equipped with a high-pressure cell. The setup allowed to investigate the effect of these process conditions on rheological properties of SBP-laccase mixtures. Rheological measurements were supported by structural investigation through light microscopy and visual observation. The feasibility of laccase-mediated foam stabilization was tested on lab scale in a high-pressure batch foaming process.

\section{Materials and methods}

\section{Materials}

Sugar beet pectin Betapec RU 301 was kindly provided by Herbstreith \& Fox KG (Neuenbürg, Germany) and used without further purification. Laccase from Trametes versicolor (CAS number: 80498-15-3) was purchased from Sigma-Aldrich (Buchs, Switzerland). The laccase was reported to have an enzymatic activity of $\geq 0.5 \mathrm{U} \mathrm{mg}^{-1}$. Phosphoric acid solution 85 wt\% (Sigma-Aldrich, Buchs, Switzerland), sodium phosphate dibasic dihydrate (Altmann Analytik, Munich, Germany), and sodium hydroxide (Merck, Darmstadt, Germany) were obtained for buffer preparation and $\mathrm{pH}$ adjustment. All samples were prepared with Milli-Q water.

\section{Solution preparation}

Sugar beet pectin and laccase solutions were prepared in $300 \mathrm{mM}$ phosphate buffer at $\mathrm{pH}$. For the preparation of SBP solutions, $2.25 \mathrm{wt} \%$ SBP powder was dissolved in phosphate buffer by stirring for at least $2 \mathrm{~h}$. If necessary, $\mathrm{pH}$ was adjusted to 5.0 adding $1 \mathrm{M}$ sodium hydroxide solution. Laccase solution was prepared at a concentration of $8 \mathrm{wt} \%$. SBP and laccase solutions were kept in an ice bath prior to experiments to ensure a temperature of $4{ }^{\circ} \mathrm{C}$ or less. For all cross-linking experiments, SBP and laccase solutions were mixed in a weight-to-volume ratio of 20 to 1 . In experiments with pure SBP solution, phosphate buffer was added instead.

\section{Steady shear and oscillatory rheology}

Steady shear rheology was performed to simulate shear applied in the high-pressure foaming process. Subsequently, oscillatory rheology was used to investigate the resulting viscoelastic properties of SBP solution cross-linked by laccase as a function of shear history. The measurements were conducted with a Modular Compact Rheometer MCR 302 (Anton Paar, Graz, Austria) equipped with a coaxial cylinder geometry CC17. The experiments comprised three main steps as illustrated in Fig. 1: (I) quick mixing of SBP and laccase, (II) shear, and (III) oscillation to measure visco-elastic response at rest. For mixing (I), $200 \mu \mathrm{L}$ laccase solution was pipetted into the CC17 cup precooled to $4^{\circ} \mathrm{C}$. Upon addition of $4 \mathrm{~g}$ SBP solution, the mixture was stirred at $4^{\circ} \mathrm{C}$ for $0.5 \mathrm{~min}$ at a shear rate of $500 \mathrm{~s}^{-1}$. The low temperature was set to reduce the number of cross-links formed during mixing. In step (II), the SBP-laccase mixture was sheared at shear rates of 5,50 and $500 \mathrm{~s}^{-1}$ for 5 to $30 \mathrm{~min}$. Within the first $5 \mathrm{~min}$ of (II), the temperature was increased from 4 to $25{ }^{\circ} \mathrm{C}$ and kept constant afterwards. Subsequently (III), the SBP-laccase mixture was oscillated at a frequency of 1 rad $\mathrm{s}^{-1}$ and a strain amplitude of $0.1 \%$ (LVR) for 60 to $120 \mathrm{~min}$ at $25{ }^{\circ} \mathrm{C}$ until an equilibrium of $G^{\prime}$ and $G^{\prime \prime}$ was reached. In order to compare results to unsheared SBP-laccase mixtures, step (II) was skipped. Instead, the temperature was increased from 4 to $25^{\circ} \mathrm{C}$ within the first $5 \mathrm{~min}$ of oscillation (III).

\section{High-pressure rheology}

In a high-pressure foaming process, gases such as carbon dioxide or nitrogen are dissolved in a continuous phase under pressure. In order to investigate the effect of pressure and the presence of dissolved $\mathrm{CO}_{2}$ on laccase-mediated cross-linking of SBP, rheological experiments were performed in a high-

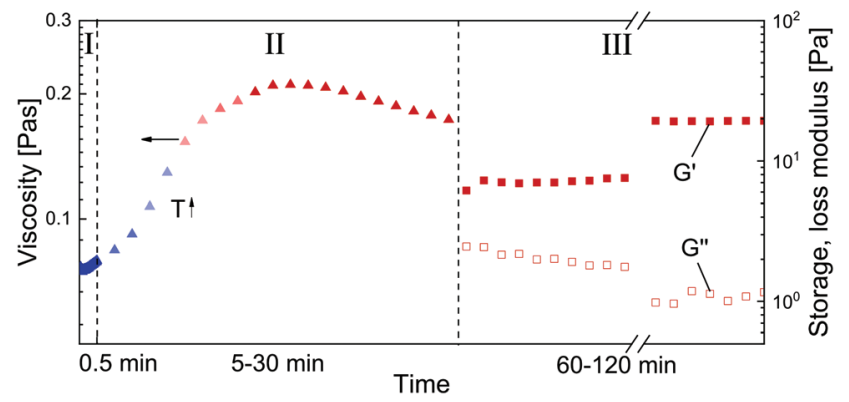

Fig. 1 Illustration of the rheological procedure: (I) mixing, (II) shearing and heating, (III) oscillating. 
pressure Couette cell CC25/Pr150 (Anton Paar, Graz, Austria) mounted on the MCR 302. The upper part of the geometry was lowered until a normal force of $\pm 0.5 \mathrm{~N}$ was reached, indicating the correct relative positioning of the magnets in the coupling. In this measuring position, air and bearing checks were conducted. In case of acceptable bearing conditions, the cell was pre-cooled to $4{ }^{\circ} \mathrm{C}$ and filled with $17.5 \mathrm{~g}$ SBP solution through the bottom inlet. To perform experiments under pressure, the bottom inlet was sealed and $\mathrm{CO}_{2}$ was supplied into the cell through the top inlet until a gauge pressure of 10 bar was reached. After closing the top inlet, a shear rate of $50 \mathrm{~s}^{-1}$ was applied for $210 \mathrm{~min}$ to reach equilibrium pressure $P_{\mathrm{eq}}$ of $8.2 \pm$ 0.4 bar above atmospheric pressure and thus saturation of SBP solution with dissolved $\mathrm{CO}_{2}$. The temperature was kept at $4{ }^{\circ} \mathrm{C}$. The laccase solution was added at rest and under pressure via the bottom inlet. $875 \mu \mathrm{L}$ laccase solution was filled into a tube connected to the bottom inlet and to the gas supply, each side closed with a valve. By lowering the pressure in the cell by 1.4 bar through the top inlet and by increasing the pressure in the laccase-filled pipe to $P_{\text {eq }}$, the laccase solution was pushed into the cell by opening the valves. The pressure re-equilibrated to $P_{\text {eq }}$. Shear was applied at a shear rate of $50 \mathrm{~s}^{-1}$ for $30 \mathrm{~min}$. As in the previous experiments, the temperature was increased from 4 to $25{ }^{\circ} \mathrm{C}$ within the first $5 \mathrm{~min}$ of shear. For experiments without laccase, buffer was added to the SBP solution instead before pressurizing the cell. For experiments at ambient pressure, the pressurization and saturation steps were skipped and the SBP solution was sheared for $10 \mathrm{~min}$ before adding the laccase solution.

\section{High-pressure batch foaming}

Feasibility of in situ cross-linking of SBP foam generated by high-pressure foaming was tested in a stirred pressure vessel (Mini bench top reactor 4569, Parr Instrument, Frankfurt, Germany). The vessel is connected to gas supply and is equipped with pressure and temperature sensors as well as a controlled pressure release valve. Temperature is controlled by a water bath connected to the double jacket of the vessel. The setup has been described in more detail by Lammers et al. ${ }^{14}$

$100 \mathrm{~g}$ SBP solution were filled into the pressure vessel kept at $25{ }^{\circ} \mathrm{C}$. After the pressure vessel was completely sealed, vacuum was drawn to reach 0.4 bar for standardization and to partly remove pre-dissolved gas from the SBP solution. $\mathrm{CO}_{2}$ was supplied to the vessel to apply a gauge pressure of 10 bar. SBP solution was stirred by a pitched blade stirrer for $20 \mathrm{~min}$ at a rotational speed of $300 \mathrm{rpm}$ to reach equilibrium pressure and saturation of the SBP solution with dissolved $\mathrm{CO}_{2} .5 \mathrm{~mL}$ laccase solution was added at rest and under pressure through the bottom inlet of the pressure vessel, according to the procedure described for the high-pressure cell of the rheometer, and mixed for either $0.5 \mathrm{~min}$ or $10 \mathrm{~min}$ at $300 \mathrm{rpm}$. Subsequently, pressure was released at a rate of $5 \mathrm{bar} \mathrm{min}^{-1}$ causing bubble nucleation and foam formation. For comparison, the process was repeated without laccase. To visualize foam expansion and collapse through the window of the HP vessel, images were taken with a CCD-camera (Mikro-NIKKOR
$55 \mathrm{~mm} 42.8$, Nikon, JP) at a distance of $1.4 \mathrm{~m}$ with a frequency of 30 images per min for expansion and 2 images per min during collapse. A non-flickering lamp was placed behind the pressure vessel for improved contrast. The point of maximum expansion was defined as the starting point of collapse.

\section{Light microscopy}

SBP-laccase mixtures were sheared in the rotational rheometer at different shear rates, according to the procedure described above, for $120 \mathrm{~min}$ to ensure equilibration of viscosity. A small amount of the sample was transferred to a microscopy slide. SBP was stained with a drop of $0.5 \%$ crystal violet solution and covered with a cover slip. After $10 \mathrm{~min}$, the sample was carefully rinsed with phosphate buffer to remove excess dye. Images were taken with a light microscope (Nikon Diaphot TMD, Nikon, Japan) at $4 \times$ magnification.

\section{Results and discussion}

Cross-linking of SBP solution by laccase was investigated rheologically under shear, pressure and presence of $\mathrm{CO}_{2}$ simulating the conditions in a high-pressure foaming process. Feasibility of in situ cross-linking of SBP in a foam was tested in a batch foaming experiment.

\section{Cross-linking under shear}

To evaluate the effect of shear on the structure formation in SBP-laccase mixtures, various steady shear conditions were applied in a rotational rheometer. Fig. 2(A) shows the viscosity under shear of SBP and laccase solution mixed at $t=0 \mathrm{~min}$. After mixing for $0.5 \mathrm{~min}$ at $4{ }^{\circ} \mathrm{C}$ and $500 \mathrm{~s}^{-1}$, shear was initiated at shear rates of 5,50 and $500 \mathrm{~s}^{-1}$ and the temperature was increased to $25^{\circ} \mathrm{C}$ over $5 \mathrm{~min}$.

For the pure SBP solution, viscosity decreases with increasing temperature and levels out at $0.035 \mathrm{mPas}$ at $25{ }^{\circ} \mathrm{C}$. For all SBP-laccase mixtures, viscosity increases immediately upon mixing of SBP and laccase solution, indicating an instant laccase-mediated cross-linking of SBP. The increase in viscosity is faster and more pronounced with decreasing shear rate. This can be attributed to a shear-induced structuring. While the SBP solution at the used concentration exhibits only slight shear-thinning behavior (see Fig. 2(B)), laccase induces the formation of cross-links, which leads to an increase in molecular size and hydrodynamic radius. Both affects the onset of shearthinning behavior. ${ }^{15}$ With higher shear rate, enhanced convection accelerates the formation of cross-links. Concurrently, alignment in the shear field is more pronounced, resulting in a lower increase in viscosity. The samples at 5 and $500 \mathrm{~s}^{-1}$ were sheared for 120 min until viscosity did not further change and were subsequently exposed to a shear ramp from 5 to $500 \mathrm{~s}^{-1}$ and vice versa. As shown in Fig. 2(B), the SBP solution cross-linked under shear at $5 \mathrm{~s}^{-1}$ is more viscous than the system cross-linked at a shear rate of $500 \mathrm{~s}^{-1}$ over the investigated range. Assuming that the intial SBP concentration as well as the number of ferulic acid groups is comparable for all 

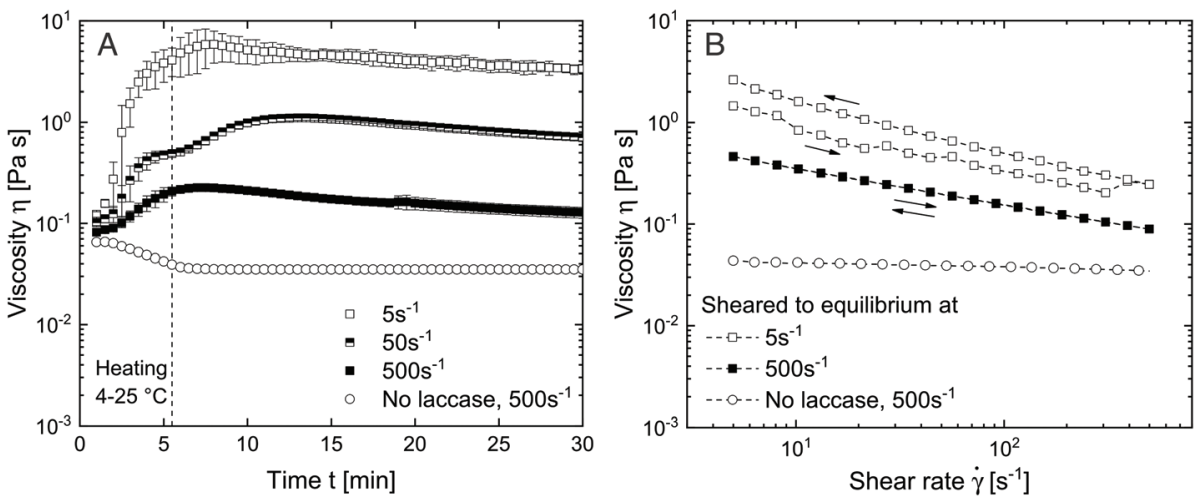

Fig. 2 (A) Viscosity $\eta$ of SBP-laccase mixtures in shear as function of shear duration $t$ at shear rates of 5,50 and $500 \mathrm{~s}^{-1}$. Temperature was kept at $4{ }^{\circ} \mathrm{C}$ for $0.5 \mathrm{~min}$ and then increased from 4 to $25^{\circ} \mathrm{C}$ over $5 \mathrm{~min}$. (B) Viscosity at $25^{\circ} \mathrm{C}$ as function of shear rate for SBP-laccase mixtures sheared at 5 $\mathrm{s}^{-1}$ and $500 \mathrm{~s}^{-1}$, respectively, until equilibrium viscosity was reached. For comparison, the viscosity of pure SBP solution is shown in (A) and (B).

samples, the higher final viscosity indicates that molecules with larger hydrodynamic radius are formed when crosslinking takes place in a weaker shear field. Hence, molecular size or branching increases with decreased shear rate. Similar processes were described by Norton et al. ${ }^{16}$ for the thermal gelation of agar solution under shear. The initial increase in viscosity upon cooling was explained by the presence of gel nuclei. The subsequent decrease in viscosity was associated with a smoothing of the gel particles. ${ }^{16}$

The samples sheared at 5 and $500 \mathrm{~s}^{-1}$ for $120 \mathrm{~min}$ until equilibrium viscosity were analyzed by light microscopy. Fig. 3(A) shows that cross-linking of SBP under slight shear results in the formation of large particles of several millimeter in size,

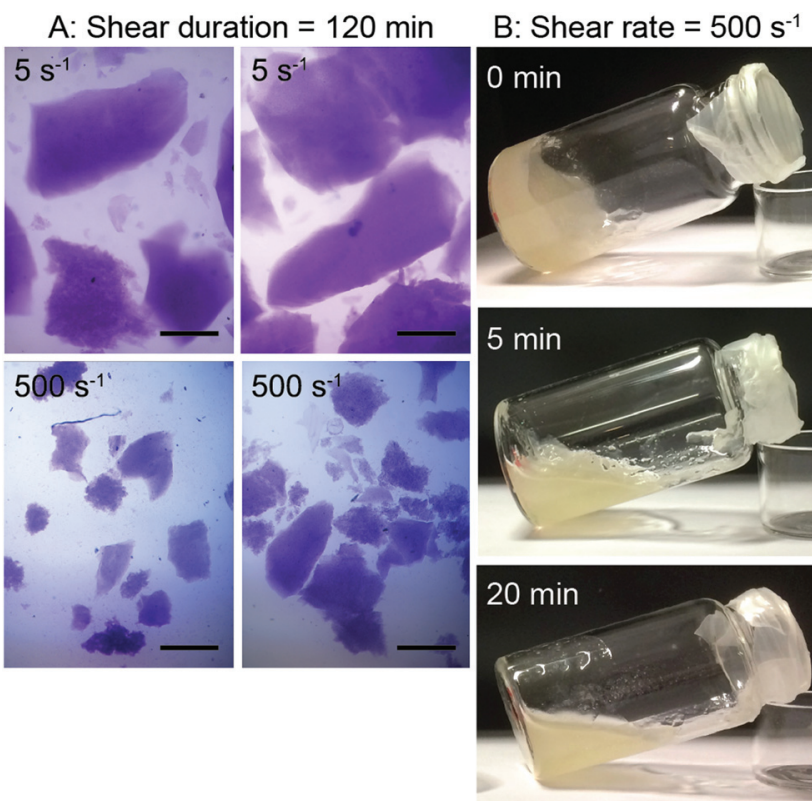

Fig. 3 (A) Light microscopy images of SBP-laccase mixtures stained with crystal violet sheared for $120 \mathrm{~min}$ at shear rates of 5 and $500 \mathrm{~s}^{-1}$. scale bar indicates $1 \mathrm{~mm}$. (B) Images of SBP-laccase mixtures $2.5 \mathrm{~h}$ after shear for 0,5 and $20 \mathrm{~min}$ at $500 \mathrm{~s}^{-1}$. whereas high shear leads to small cross-linked particles suspended in SBP solution. This is in line with the difference in hydrodynamic radius suggested for the viscosity measurements.

The formation of gel particles if gelling occurs under shear is well-known from production of microgels, also referred to as broken or fluid gels. ${ }^{17}$ Norton et al. ${ }^{18}$ found that smaller gel particles are being formed when applying higher shear rates. However, the particles are in the micrometer range and thus not comparable in size with the large particles formed in this study. Several other publications discuss the formation of differently shaped gel particles controlled through shear stresses and liquid phase viscosity ${ }^{19,20}$ and their application, e.g., to mimic fat droplets or starch granules. ${ }^{21}$ As this study focuses on the stabilization of continuous phases, the formation of suspensions with small gel particles is not desired. As a shear duration of $120 \mathrm{~min}$ is furthermore not relevant for a high-pressure foaming process, the effect of shorter shear durations was assessed. Samples were taken after mixing and shearing at $500 \mathrm{~s}^{-1}$ for 0,5 or $20 \mathrm{~min}$ and stored for $120 \mathrm{~min}$. Visual analysis in Fig. 3(B) reveals that the non-sheared sample forms a gel, $5 \mathrm{~min}$ of shear leads to the formation of a broken, fluid gel, and after $20 \mathrm{~min}$ of shear, laccase turns the SBP solution into a liquid suspension with small gel particles.

To investigate visco-elastic properties of SBP-laccase mixtures sheared for different durations, steady shear was stopped after 5 to $30 \mathrm{~min}$. Subsequently, an oscillatory time sweep was conducted at constant strain amplitude and frequency until equilibrium of storage modulus $G^{\prime}$ and loss modulus $G^{\prime \prime}$ was reached (III). Average $G^{\prime}$ and $G^{\prime \prime}$ in equilibrium are plotted as a function of preceding shear duration in Fig. 4(A) and (B), respectively. For the non-sheared system, SBP and laccase solutions were only mixed for $0.5 \mathrm{~min}$ followed by oscillation. The final storage modulus decreases exponentially with increasing shear duration for all applied shear rates.

The decrease in shear from 500 to 50 and $5 \mathrm{~s}^{-1}$ leads to a weaker dependency of $G^{\prime}$ on shear duration. $G^{\prime \prime}$ is not affected by shear duration at highest shear rate but increases if the mixture is moderately or slowly sheared during cross-linking. The increase in ratio of loss to storage modulus $G^{\prime \prime} / G^{\prime}$ with 

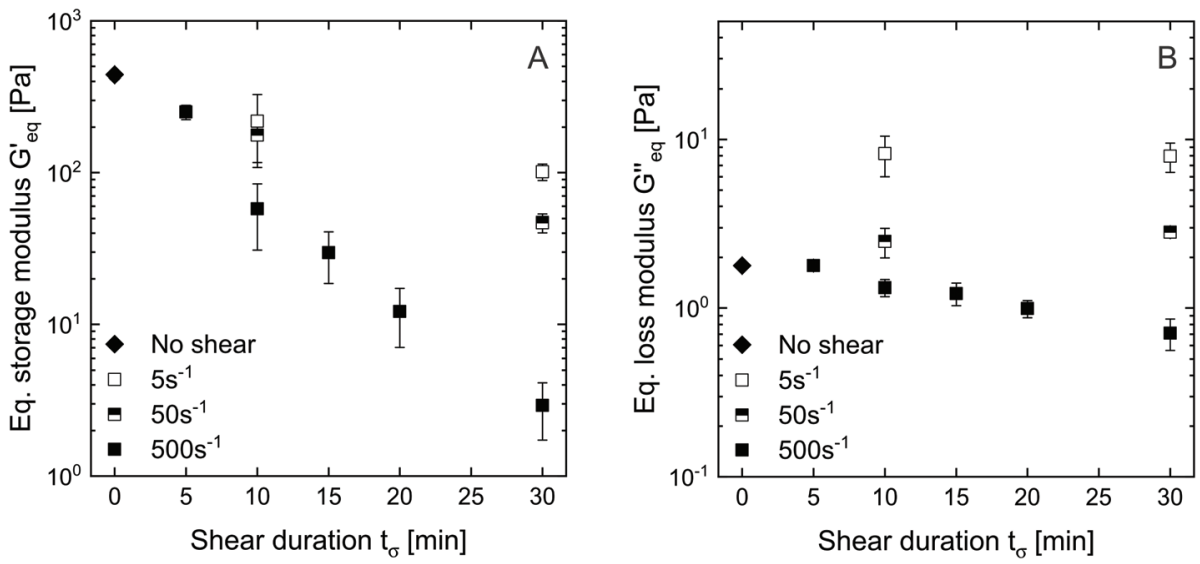

Fig. 4 Equilibrium storage modulus (A) and loss modulus (B) of SBP-laccase mixtures as function of shear duration applied in step (II) at shear rates of 5,50 and $500 \mathrm{~s}^{-1}$.

increasing shear rate and shear duration implies a decrease in structural strength. ${ }^{22}$ While stiff SBP gels are obtained if laccase-mediated cross-linking occurs at rest, application of shear causes gel fluidization. As described above, shear induced the formation of cross-linked SBP particles. If the shear duration is shorter than the time needed to cross-link all available ferulic acid groups, cross-linking continues at rest. The results suggest that this allows the formation of crosslinks in between formed particles, as illustrated in Fig. 5. Hence, a shorter shear duration results in the formation of a more coherent structure exhibiting a higher storage modulus. As a lower shear rate generates larger gel particles, friction between particles and thus $G^{\prime \prime}$ is increased. However, longer shear duration does not seem to affect these friction planes. These findings may be of interest for injectable, in situ

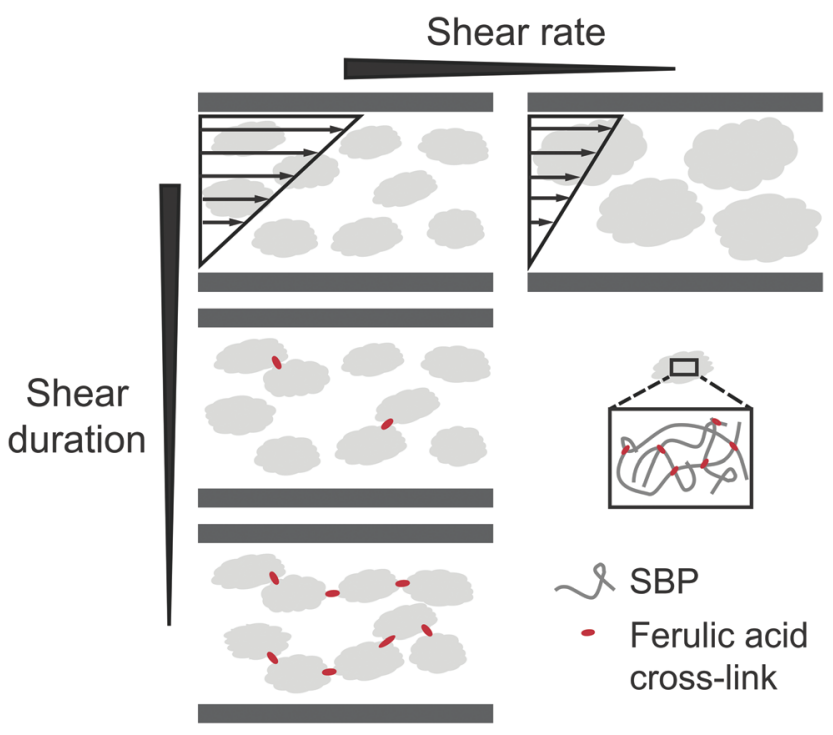

Fig. 5 Illustration of the proposed effect of shear rate and shear duration on laccase-mediated cross-linking of sugar beet pectin in solution. forming hydrogels, e.g., for biomedical applications. ${ }^{23,24}$ Here, the flow conditions in the syringe may be optimized to yield a chemically or enzymatically cross-linked hydrogel with aimed structure and mechanical properties.

\section{Cross-linking at presence of carbon dioxide}

In high-pressure foaming, a gas, e.g., $\mathrm{CO}_{2}$ used in this study, is dissolved in the continuous phase under pressure. In order to integrate laccase-mediated cross-linking in a highpressure foaming process, it has to be considered that the oxidation of ferulic acid requires the presence of oxygen. ${ }^{4}$ Addition of $\mathrm{CO}_{2}$ might reduce the contact area between solution and oxygen in the head space and thus affect oxidation. To investigate the effect of $\mathrm{CO}_{2}$, the viscosity of the SBP solution was assessed under pressure and steady shear during saturation with $\mathrm{CO}_{2}$ and addition of laccase. Fig. 6 shows the viscosity of the SBP solution with and without supply of $\mathrm{CO}_{2}$

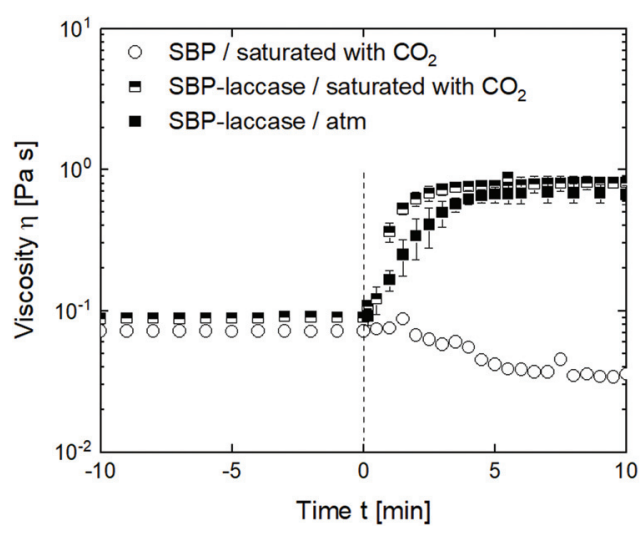

Fig. 6 Viscosity of SBP solution and SBP-laccase mixtures at a gauge pressure of 8.5 bar exerted by $\mathrm{CO}_{2}$ or at atmospheric pressure: at $<0 \mathrm{~min}$, samples are sheared at $\dot{\gamma}=300 \mathrm{~s}^{-1}$ and $T=4{ }^{\circ} \mathrm{C}$ for $\mathrm{CO}_{2}$ dissolution; at $t=0 \mathrm{~min}$, laccase is added; at $t>0 \mathrm{~min}$, samples are sheared at $\dot{\gamma}=50 \mathrm{~s}^{-1}$ and temperature is increased to $25^{\circ} \mathrm{C}$ for SBP cross-linking. 
and laccase. At $<0 \mathrm{~min}$, the SBP solution was sheared at 300 $\mathrm{s}^{-1}$ and $4{ }^{\circ} \mathrm{C}$ either at atmospheric pressure or at 8.5 bar above atmospheric pressure exerted by $\mathrm{CO}_{2}$. Shear and pressure cause dissolution of $\mathrm{CO}_{2}$ in the SBP solution. Neither pressure nor dissolved $\mathrm{CO}_{2}$ affect the viscosity of the pure SBP solution at the studied conditions. At $t=0 \mathrm{~min}$, laccase is added into two samples, the shear rate is reduced to $50 \mathrm{~s}^{-1}$ and the temperature is increased from 4 to $25{ }^{\circ} \mathrm{C}$. The viscosity of SBP solution without laccase increases slightly due to the lower shear rate, followed by a temperature-induced decrease in viscosity. The outliers might result from bubble nucleation as the temperature increases or from the limited sensitivity of the magnetic coupling of the highpressure setup. With addition of laccase, the viscosity increases similarly for the sample with added $\mathrm{CO}_{2}$ compared to the sample at atmospheric pressure and reaches a level similar to the results shown in Fig. 2 at a shear rate of 50 $\mathrm{s}^{-1}$. This indicates that there is sufficient oxygen in the SBP solution to oxidize the ferulic acid.

\section{Integration of cross-linking in a high-pressure batch foaming process}

SBP foams were generated with and without laccase in a highpressure vessel and foam collapse was observed. For this purpose, the SBP solution was saturated with $\mathrm{CO}_{2}$ under shear and a gauge pressure of 8.5 bar. Similar to the experiments in the high-pressure cell of the rheometer, laccase was added under pressure and mixed for $0.5 \mathrm{~min}$. Subsequently, mixing was either continued for $9.5 \mathrm{~min}$ or the mixture was immediately depressurized. For comparison, pure SBP foams were produced accordingly without laccase.

Fig. 7 shows images of (A) pure SBP foam, (B) SBP-laccase foam mixed for $0.5 \mathrm{~min}$, and (C) SBP-laccase foam mixed for $10 \mathrm{~min}$ prior to depressurization over $60 \mathrm{~min}$ after maximum expansion. Pure SBP solution is clearly foaming but bubbles grow and collapse rapidly after expansion. The foaming or emulsification ability of sugar beet pectin has been elucidated by several studies: the proteinaceous moiety in sugar beet pectin as well as the hydrophobic character of ferulic acid are thought to play a major role in interfacial stabilization. ${ }^{25,26}$ Nevertheless, at the concentrations used in this study, their stabilization ability seems to be limited.

The addition of laccase and a mixing time of $0.5 \mathrm{~min}$ results in less foam collapse, however, images show clear bubble growth (Fig. 7(B)). When laccase is mixed with SBP solution for $10 \mathrm{~min}$ prior to depressurization, the resulting foam collapses only slightly within $60 \mathrm{~min}$ after expansion. Bubble size increases within the first $30 \mathrm{~min}$, after which destabilization seems to slow down (Fig. 7(C)). The bubble coarsening was not quantified as the visible bubbles are in contact with the window and might thus not be representative for the whole foam volume.

The laccase addition significantly increased the stability and decreased collapse and bubble growth in SBP foams. As SBP foam without laccase is highly unstable, laccase-mediated

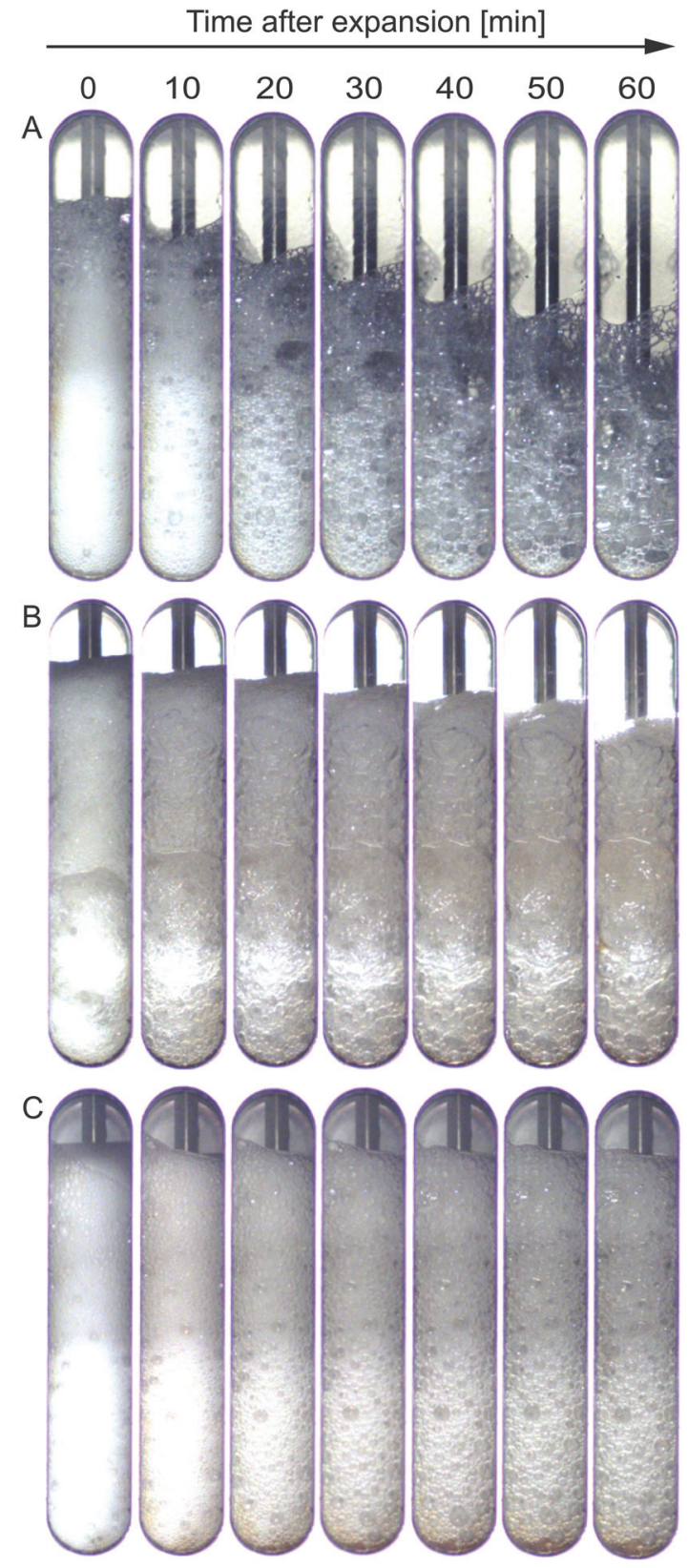

Fig. 7 Images of (A) SBP foam without laccase, (B) SBP-laccase foam mixed for $0.5 \mathrm{~min}$ prior to depressurization, and (C) SBP-laccase foam mixed for $10 \mathrm{~min}$ prior to depressurization over $60 \mathrm{~min}$ in the highpressure vessel. Time point $t=0 \mathrm{~min}$ corresponds to the point of maximum expansion. The window width equals $16.5 \mathrm{~mm}$.

cross-linking of SBP does not allow to instantly prevent foam destabilization, as qualitatively illustrated in Fig. 8(A). Instead, if cross-linking happens partly prior to depressurization, the formed cross-linked SBP gel bodies stabilize the foam immediately through an increase in continuous phase viscosity (Fig. 8 (B)). As cross-linking continues at rest if shear is applied for only $10 \mathrm{~min}$, as shown in the results at different shear durations, SBP gel bodies interconnect in the foam lamellae resulting in improved stability. 
A: Short mixing time

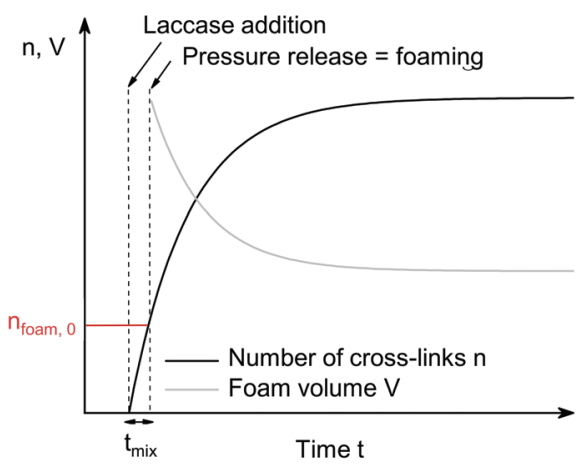

$\mathrm{t}_{\mathrm{mix}}$ short $=\mathrm{n}$ low $=$ unstable foam

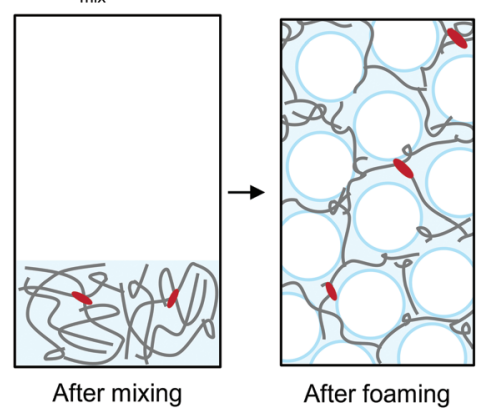

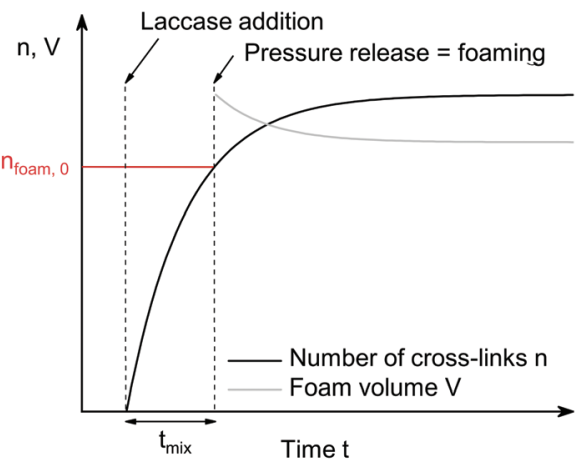

$\mathrm{t}_{\text {mix }}$ long $=\mathrm{n}$ high $=$ stable foam

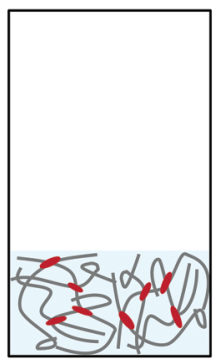

After mixing

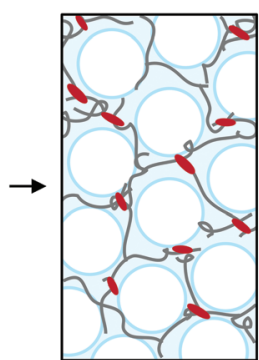

After foaming

\& SBP - Ferulic acid cross-link

Foam bubble

Fig. 8 Schematic graph and illustration of cross-linking and foam collapse kinetics at (A) short and (B) long mixing times prior to pressure release. $n$ is the number of ferulic acid cross-links. $n_{\mathrm{foam}, 0}$ is the number of cross-links in the foam immediately after pressure release. The bubble size is depicted to be constant for simplicity.

\section{Conclusions}

This study has demonstrated that laccase-mediated crosslinking can be integrated into a high-pressure foaming process to increase foam stability of sugar beet pectin foam. The results have shown that carbon dioxide and pressure do not hinder cross-linking of sugar beet pectin at the investigated conditions. However, shear applied during cross-linking causes the formation of cross-linked particles suspended in a liquid phase rather than a coherent gel. The size of these particles decreases with increasing shear rate resulting in a lower viscosity of the SBP solution after cross-linking. Terminating shear before all available ferulic acid groups have been crosslinked permits the formation of further cross-links at rest, resulting in a structure with higher network strength. This has to be considered for the alignment of laccase addition and foam generation in a high-pressure foaming process. If laccase is added too early in the process, a suspension of gel particles might form, which does not provide sufficient stabilization of the continuous phase. However, if laccase is added only shortly before foam generation, the number of formed crosslinks is not yet sufficient to prevent the highly unstable sugar beet pectin foam from collapsing, as shown experimentally. Thus, to optimize laccase-induced stabilization of SBP foam, it is crucial to align well the kinetics of cross-linking with the kinetics of shear-induced structuring, foam generation and foam collapse.

\section{Conflicts of interest}

This study has been supported by Nestlé Research Lausanne.

\section{Acknowledgements}

The authors thank Herbstreith \& Fox KG for providing sugar beet pectin and Peter Fischer, Antoni Sánchez-Ferrer and Simon Kuster from ETH Zürich for their valuable advice.

\section{References}

1 G. M. Campbell and E. Mougeot, Creation and characterisation of aerated food products, Trends Food Sci. Technol., 1999, 10, 283-296.

2 B. Zeeb, L. Fischer and J. Weiss, Stabilization of food dispersions by enzymes, Food Funct., 2014, 5, 198-213.

3 B. Zeeb, D. J. McClements and J. Weiss, Enzyme-based strategies for structuring foods for improved functionality, Annu. Rev. Food Sci. Technol., 2017, 8, 21-34. 
4 C. F. Thurston, The structure and function of fungal laccases, Microbiology, 1994, 140, 19-26.

5 R. C. Minussi, G. M. Pastore and N. Durán, Potential applications of laccase in the food industry, Trends Food Sci. Technol., 2002, 13, 205-216.

6 V. Micard and J.-F. Thibault, Oxidative gelation of sugarbeet pectins: use of laccases and hydration properties of the cross-linked pectins, Carbohydr. Polym., 1999, 39, 265273.

7 F. Littoz and D. J. McClements, Bio-mimetic approach to improving emulsion stability: cross-linking adsorbed beet pectin layers using laccase, Food Hydrocolloids, 2008, 22, 1203-1211.

8 B. Zeeb, M. Gibis, L. Fischer and J. Weiss, Crosslinking of interfacial layers in multilayered oil-in-water emulsions using laccase: characterization and pH-stability, Food Hydrocolloids, 2012, 27, 126-136.

9 S. Soltani and A. Madadlou, Gelation characteristics of the sugar beet pectin solution charged with fish oilloaded zein nanoparticles, Food Hydrocolloids, 2015, 43, 664-669.

10 S. Soltani and A. Madadlou, Two-step sequential crosslinking of sugar beet pectin for transforming zein nanoparticle-based pickering emulsions to emulgels, Carbohydr. Polym., 2015, 136, 738-743.

11 A. Einstein, Über die von der molecularkinetischen Theorie der Wärme geforderte Bewegung von in ruhenden Flussigkeiten suspendierten Teilchen, Annalen der Physik, 1905, 322, 549-560.

12 S. T. Lee and C. B. Park, Foam extrusion: principles and practice, foam extrusion: principles and practice, CRC Press, 2014.

13 P. Sepulveda and J. G. Binner, Processing of cellular ceramics by foaming and in situ polymerisation of organic monomers, J. Eur. Ceram. Soc., 1999, 19, 2059-2066.

14 V. R. G. Lammers, A. Morant, J. Wemmer and E. J. Windhab, High-pressure foaming properties of carbon dioxide-saturated emulsions, Rheol. Acta, 2017, 56, 841850 .
15 P. A. Williams and G. O. Phillips, Introduction to food hydrocolloids, in Handbook of hydrocolloids, CRC Press, 2009.

16 I. T. Norton, D. A. Jarvis and T. J. Foster, A molecular model for the formation and properties of fluid gels, Int. J. Biol. Macromol., 1999, 26, 255-261.

17 T. Farjami and A. Madadlou, Fabrication methods of biopolymeric microgels and microgel-based hydrogels, Food Hydrocolloids, 2017, 62, 262-272.

18 I. Norton, T. Foster and R. Brown, The science and technology of fluid gels, in Gums and Stabilisers for the Food Industry 9, ed. P. A. Williams and G. O. Philips, Elsevier, 1998.

19 B. Wolf, W. J. Frith, S. Singleton, M. Tassieri and I. T. Norton, Shear behaviour of biopolymer suspensions with spheroidal and cylindrical particles, Rheol. Acta, 2001, 40, 238-247.

20 B. Wolf, W. J. Frith and I. T. Norton, Influence of gelation on particle shape in sheared biopolymer blends, J. Rheol., 2001, 45, 1141-1157.

21 D. J. McClements, C. Chung and B. Cheng Wu, Structural design approaches for creating fat droplet and starch granule mimetics, Food Funct., 2017, 8, 498-510.

22 T. G. Mezger, The rheology handbook: for users of rotational and oscillatory rheometers, Vincentz Network GmbH \& Co KG, 2006.

23 S. Hou, R. Lake, S. Park, S. Edwards, C. Jones and K. J. Jeong, Injectable macroporous hydrogel formed by enzymatic cross-Linking of gelatin microgels, ACS Appl. Bio Mater., 2018, 1, 1430-1439.

24 B. Balakrishnan and A. Jayakrishnan, Self-cross-linking biopolymers as injectable in situ forming biodegradable scaffolds, Biomaterials, 2005, 26, 3941-3951.

25 P. A. Williams, C. Sayers, C. Viebke, C. Senan, J. Mazoyer and P. Boulenguer, Elucidation of the emulsification properties of sugar beet pectin, J. Agric. Food Chem., 2005, 53, 3592-3597.

26 C. K. Siew and P. A. Williams, Role of protein and ferulic acid in the emulsification properties of sugar beet pectin, J. Agric. Food Chem., 2008, 56, 4164-4171. 